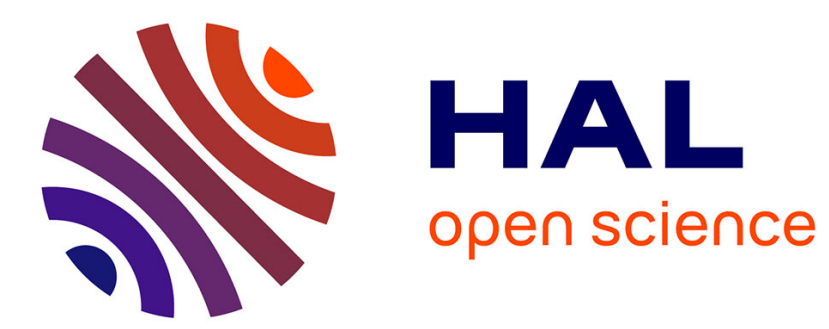

\title{
LE PRENOM DE L'ENFANT OU LA FANTAISIE DES PARENTS
}

\author{
Maxime Péron
}

\section{To cite this version:}

Maxime Péron. LE PRENOM DE L'ENFANT OU LA FANTAISIE DES PARENTS. Revue juridique de l'Ouest , 2016, 3, pp.7. hal-02010677

\section{HAL Id: hal-02010677 \\ https://hal.univ-brest.fr/hal-02010677}

Submitted on 7 Feb 2019

HAL is a multi-disciplinary open access archive for the deposit and dissemination of scientific research documents, whether they are published or not. The documents may come from teaching and research institutions in France or abroad, or from public or private research centers.
L'archive ouverte pluridisciplinaire HAL, est destinée au dépôt et à la diffusion de documents scientifiques de niveau recherche, publiés ou non, émanant des établissements d'enseignement et de recherche français ou étrangers, des laboratoires publics ou privés. 


\title{
LE PRENOM DE L'ENFANT OU LA FANTAISIE DES PARENTS
}

\author{
Par \\ Maxime PERON \\ Doctorant en droit privé, CRDP (EA 3881), Université de Bretagne Occidentale, Universidade de Sao Paulo.
}

Entre parents, le choix du prénom fait l'objet d'intenses rêveries et négociations ${ }^{1}$, pouvant aboutir sur un choix audacieux, ayant pour conséquence fantaisie ou bizarrerie.

Alors, qu'en 2015, les parents ont privilégié les prénoms Louise et Léo et délaissé les prénoms Pétunia, Lotus, Térébenthine ou Périclès, certains préfèrent choisir des prénoms fantaisistes mais pouvant porter atteinte à l'intérêt de l'enfant. Tel est le cas lorsqu'ils choisissent le prénom Léo-pard².

Lorsqu'ils privilégient la fantaisie, les parents peuvent oublier le rôle d'identification du prénom. Pour identifier une personne, il faut dégager les éléments qui permettent de cerner son identité afin de l'individualiser dans la société ${ }^{3}$. La loi a organisé l'état civil afin de permettre à la société d'identifier ses sujets ${ }^{4}$. L'un de ses éléments d'identification est le nom, qui comprend deux éléments : le nom de famille et le prénom ${ }^{5}$. Parfois qualifié « d'accessoire décoratif $»^{6}$, le prénom reste un accessoire nécessaire et obligatoire ${ }^{7}$ pour permettre d'individualiser un individu au sein de sa famille ${ }^{8}$ et doit être indiqué dans l'acte de naissance ${ }^{9}$. Le choix du prénom revient aux parents, mais ce choix peut s'avérer difficile : quel prénom choisir ?

Alors que lors du choix du prénom, les parents peuvent laisser libre court à leur imagination à travers un choix fantaisiste (I), cette fantaisie peut s'avérer problématique pour son porteur, justifiant son changement (II).

\section{I - L'ATTRIBUTION DU PRENOM OU LA FANTAISIE DES PARENTS}

Tant l'étude des règles législatives (A) que l'analyse de la jurisprudence (B) démontrent l'ampleur de la liberté accordée aux parents lors du choix des prénoms de l'enfant, liberté pouvant aboutir à un choix fantaisiste.

\section{A - Un libéralisme accordé par le législateur}

En principe, les prénoms de l'enfant sont choisis par ses père et mère ${ }^{10}$, ce qui en fait un attribut de l'autorité parentale ${ }^{11}$.

Pendant longtemps, la législation en la matière était restrictive ${ }^{12}$, l'article $1^{\text {er }}$ de la loi du 11 germinal an XI imposant de choisir le prénom «soit dans les différents calendriers », soit parmi les prénoms « des personnages connus dans l'histoire ancienne ». Si ces conditions n'étaient pas remplies, l'officier de l'état civil pouvait refuser

\footnotetext{
${ }^{1}$ F. BELLIVIER, Droit des personnes, Issy-les-Moulineaux, LGDJ-Lextenso, 2015, p. 78, n 59.

${ }^{2}$ CA Rennes, 25 janv. 2016, n¹5/01705, JurisData n 2016-001207 : Dr. Fam. 2016, n 3, comm. 55, obs. A.-C. Réglier.

${ }^{3}$ A. MARAIS, Droit des personnes, Paris, Dalloz, 2e éd., 2014, p. 73, nº 98.

${ }^{4}$ Ibid., p. $74, \mathrm{n}^{\circ} 99$.

${ }^{5}$ Ibid., p. $76, \mathrm{n}^{\circ} 103$.

${ }^{6}$ J. HAUSER, obs. RTD civ. 1993. 559.

${ }^{7}$ F. TERRE et D. FENOUILleT, Droit civil : les personnes, Paris, Précis Dalloz, 8e éd. 2012, p. 190, n 189.

${ }^{8}$ A. BATTEUR, Droit des personnes, des familles et des majeurs protégés, Issy-les-Moulineaux, LGDJ-Lextenso, 8e éd. 2015, p. 55, n 89 ; A. MARAIS, Droit des personnes, op. cit., p. 99, n 136 ; B. TEYSSIE, Droit civil : les personnes, Paris, LexisNexis, 17e éd., 2015, p. 227, n 331 ; F. TERRE et D. FENOUILLET, Droit civil, op. cit., p. 190, n 189 ; J. CARBONNIER, Droit civil, Paris, PUF, Quadrige, 2004, p. 437, n 229 ; P. BONFILS et A. GOUTTENOIRE, Droit des mineurs, Paris, Précis Dalloz, 2e éd., 2014, p. 307, n 475 ; G. MARTY et P. RAYNAUD, Droit civil, Paris, Sirey, 3e éd., 1976, p. 820, nº 728.

${ }^{9}$ L. 6 fructidor an II, art. $1^{\text {er }}$; art. 57 al. $1^{\text {er }}$ C. civil.

${ }^{10}$ Art. 57, al. 2 C. civ.

${ }^{11}$ B. TEYSSIE, Droit civil, op. cit., p. 228.

12 A. MARAIS, Droit des personnes, op. cit., p. 99, $\mathrm{n}^{\circ} 138$.
} 
d'inscrire le prénom. Ainsi, un enfant pouvait être prénommé Napoléon ${ }^{13}$ ou Cochon ${ }^{14}$. Cette rigidité de la loi s'est progressivement estompée au profit d'un libéralisme grandissant; d'abord par une instruction ministérielle de 1966, enjoignant aux officiers de l'état civil de ne refuser que les prénoms de pure fantaisie susceptibles de nuire aux intérêts de l'enfant ${ }^{15}$; ensuite, par la loi du 8 janvier 1993 qui a consacré le libéralisme en matière de choix des prénoms, en abrogeant la loi de l'an XI.

Néanmoins, cette liberté reste contrôlée, l'officier de l'état civil, bien que tenu de transcrire les prénoms du déclarant ${ }^{16}$, si le choix lui apparait contraire à l'intérêt de l'enfant ou aux droits des tiers à voir protéger leur nom de famille, en avisera le procureur de la République, qui, saisira lui-même le juge aux affaires familiales s'il juge la démarche fondée ${ }^{17}$. Ce dernier pourra ordonner la suppression du prénom des registres de l'état civil ${ }^{18}$. Il en va de même lorsque le prénom est incompatible avec un autre prénom ou avec le nom de famille.

Alors, les parents seront invités à modifier leur choix, et à défaut, le juge attribuera lui-même un autre prénom et dont la mention sera portée en marge des actes de l'état civil de l'enfant. L'appréciation du choix du prénom, relevant du pouvoir souverain des juges du fond ${ }^{19}$, et nécessitant de prendre en compte l'intérêt de l'enfant, conforte le libéralisme.

\section{B - Un libéralisme conforté par la jurisprudence}

De l'observation des quelques décisions jurisprudentielles sur le choix des prénoms de l'enfant, deux constats peuvent être soulevés : d'une part, la fantaisie n'est pas automatiquement sanctionnée par le juge, d'autre part, pour accepter ou refuser un prénom, il est pris en compte sa consonance commerciale, son caractère de diminutif ou sa réduction à des initiales, une orthographe particulière, ou encore sa consonance ridicule ou/et préjudiciable.

Ainsi, la consonance commerciale du prénom Nutella a justifié son refus, tandis que les prénoms Mickey ${ }^{20}$ et Chanel $^{21}$ ont été acceptés. Alors qu'un prénom ne peut être réduit aux initiales MJ, le caractère de diminutif du prénom Kinou a justifié son refus ${ }^{22}$, tandis que Dany, diminutif de Danielle, a été accepté23.

L'appréciation de l'orthographe du prénom par le juge est quant à elle variable ${ }^{24}$. Ainsi, elle a pu être très libérale en acceptant la requête des parents de rectifier Mathieu en Mattieu au motif que c'était la véritable écriture du nom de l'évangéliste et le prénom de baptême de l'enfant ${ }^{25}$, ou au contraire plus stricte en refusant le prénom Marti avec un accent aigu sur le $i$, au motif que les registres de l'état civil doivent être tenus exclusivement en français, langue constitutionnellement officielle du pays, laquelle ignore l'accent aigu sur les $\mathrm{i}^{26}$.

Par ailleurs, s'appuyant sur le respect de l'intérêt de l'enfant, le juge apprécie le prénom en fonction de sa consonance ridicule, ou de son caractère préjudiciable pour son porteur. Parfois, le juge a sanctionné la fantaisie des parents en refusant les prénoms Titeuf ${ }^{27}$, Léo-Pard, de Babord et Tribord pour des jumeaux ${ }^{28}$, de Joyeux et Patriste pour un même enfant ${ }^{29}$ ou encore de Fraise alors que Cerise est accepté ${ }^{30}$. Parfois, des prénoms, à

\footnotetext{
${ }^{13}$ A. BATTEUR, Droit des personnes, des familles et des majeurs protégés, op. cit., p. 55, n 89.

${ }^{14}$ F. LAROCHE-GISSEROT, "Le choix du prénom", AJ famille, 2012, 306.

${ }^{15}$ Instruction ministérielle du 12 avril 1966, modifiant l'instruction générale relative à l'état civil, JO 3 mai 1966 ; voir R. NERSON, RTD civ., 1966. 522.

${ }_{16}$ Art. 57, al. 2 C. civ.

${ }^{17}$ Art. 57, al. 3 C. civ.

18 Art. 57, al. 4 C. civ.

${ }^{19}$ Cass., Civ. 1 $1^{\mathrm{e}}, 15$ févr. 2012 : D. 2012. 2267. obs. P. BONFILS et A. GOUTTENOIRE; RTD civ. 2012. 287, obs. J. HAUSER ; RJPF 2012-4/14, obs. I. CORPART.

${ }^{20}$ CA Douai, 15 mai 2000, RG n ${ }^{\circ}$ 1999/01445, Juris-Data ${ }^{\circ} 116096$.

${ }^{21}$ CA Aix-en-Provence, 24 mai 1988, RG n 87/7129, Juris-Data n 046248 et TGI Rochefort, 7 mars 1990, JurisData ${ }^{\circ} 042279$.

${ }^{22}$ CA Aix-en-Provence, 9 mars 2007, RG n 06/12622, Juris-Data ${ }^{\circ} 342861$.

${ }^{23}$ CA Toulouse, 4 avr. 2006, RG n 05/06548, Juris-Data ${ }^{\circ} 304859$.

${ }^{24}$ F. LAROCHE-GISSEROT, "Le choix du prénom", AJ famille, 2012, 306.

${ }^{25}$ CA Toulouse, 9 mai 1989, RG n ${ }^{\circ}$ 670/89, Juris-Data ${ }^{\circ} 048934$.

${ }^{26}$ CA Montpellier, 26 nov. 2001, RG n 01/02858: Dr. fam. oct. 2002, p. 27, obs. P. MURAT.

${ }^{27}$ CA Versailles, 7 oct. 2010 : AJ famille 2011. 53, obs. CHENEDE ; Cass., civ. $1^{\mathrm{e}}, 15$ févr. 2012, Bull. civ. I., $\mathrm{n}^{\circ}$ 32 : D. 2012. 552 ; AJ famille 2012. 231, obs. LAMBERT ; RTD civ. 2012. 287 obs. HAUSER ; Gaz. pal. 2012. 819, note PIERRROUX.

${ }^{28}$ F. LAROCHE-GISSEROT, art. cit.

${ }^{29}$ CA Montpellier, 4 oct. 2006, RG n 05/1307 : JCP 2007, IV, 1944.

${ }^{30}$ TGI Valenciennes 19 janv. 2015.
} 
l'apparence fantaisiste, mais surtout préjudiciables, sont acceptés comme Canta $^{31}$, choisi en hommage au chanteur devenu meurtrier, au motif que sa célébrité n'est pas destinée à perdurer dans les mémoires et que le prénom a une consonance latine évoquant le chant.

Enfin, l'association ridicule entre le prénom et le nom, ou entre deux prénoms, peut être refusée par le juge. L'exemple le plus connu, pourtant admis, est celui de l'association entre le prénom Mégane précédant le nom Renaud $^{32}$, les juges du fond estimant que l'allusion passerait bientôt inaperçue au rythme où changent les modèles automobiles.

Il résulte de ces exemples que la fantaisie des parents est largement admise, la loi laissant le juge maître de décider, sans gage de cohérence. Cette incohérence peut avoir comme remède le changement ultérieur du prénom.

\section{II - LE CHANGEMENT DE PRENOM OU LE REMEDE A LA FANTAISIE DES PARENTS}

Lorsque le prénom ne va pas, il faut le changer ${ }^{33}$. Remède à la fantaisie des parents, l'encadrement légal du changement de prénom (A) accorde un rôle de contrôle au juge (B).

\section{$A$ - Un remède encadré par le législateur}

Rare $^{34}$, le changement de prénom est strictement encadré, la loi du 6 fructidor an II, toujours en vigueur, disposant qu'aucun citoyen ne peut porter de prénom autre que celui exprimé dans son acte de naissance. Aucune procédure administrative n'a été prévue, posant une immutabilité rigoureuse ${ }^{35}$.

Ce principe d'immutabilité a d'abord été assoupli pour deux hypothèses particulières que sont l'adoption ${ }^{36}$ et la francisation du prénom en cas d'acquisition de la nationalité française ${ }^{37}$. Concernant la première hypothèse, le changement est envisageable à la fois pour l'adoption plénière ${ }^{38}$ et l'adoption simple ${ }^{39}$, à la demande du ou des adoptants, et a pour objectif de renforcer le lien que l'adoption crée avec l'adopté 40 .

Ce principe s'est ensuite assoupli par la loi n 55-1465 du 12 novembre 1955 en permettant le changement de prénom à la suite d'une procédure judiciaire. En ce sens, l'article 60 du Code civil dispose que toute personne qui justifie d'un intérêt légitime peut demander à changer de prénom. En la matière, c'est le juge aux affaires familiales qui est compétent, mais la demande est à l'initiative de l'intéressé lui-même ou de son représentant s'il est mineur ${ }^{41}$. Dans ce dernier cas, l'enfant de plus de treize ans devra consentir à la modification de son prénom.

Du fait de son imprécision, la notion d'intérêt légitime est appréciée aléatoirement par la jurisprudence ${ }^{42}$. Nécessairement apprécié in concreto et non en fonction d'un motif d'ordre général ${ }^{43}$, le contrôle de cette notion par le juge pourra panser les plaies de la fantaisie des parents.

\section{B - Un remède contrôlé par la jurisprudence}

\footnotetext{
${ }^{31}$ CA Bordeaux, 22 oct. 2009 : Dr. fam. 2009, focus n 1, obs. M. LAMARCHE.

${ }^{32}$ CA Rennes, 4 mai 2000 : JCP 2001, IV, 2655.

33 I. CORPART, "Changement de prénom", AJ famille 2012, 311.

${ }^{34}$ L. BRIAND, «Appréciation par les juridictions de l'intérêt légitime à la modification du prénom », AJ famille 2012. 316 ; B. COULMONT, Sociologie des prénoms, Paris, La découverte, 2014, p. 27.

${ }^{35}$ F. TERRE et D. FENOUILLET, Droit civil, op. cit., p. 192, n 192.

${ }^{36}$ Loi n ${ }^{\circ} 49-572$ du 23 avril 1949.

${ }^{37}$ Loi n ${ }^{\circ} 72-964$ du 25 oct. 1972 (art. 1 et 2) ; J. BOUTON et G. AUVELA, « Des prénoms francisés », in mélanges D. Huet-Weiller, PU Strasbourg - LGDJ, 1994, p. 27.

${ }^{38}$ Art. 357, al. 2 C. civ.

${ }^{39}$ Loi $^{\circ}$ 2011-1862 du 13 déc. 2011 relative à la répartition des contentieux et à l'allégement de certaines procédures juridictionnelles.

${ }^{40}$ P. BONFILS et A. GOUTTENOIRE, Droit des mineurs, op. cit., p. 312, $\mathrm{n}^{\circ} 180$.

${ }^{41}$ G. HILGER, «L'office du juge et le prénom », $L P A, 13$ juin $2013, \mathrm{n}^{\circ} 118$, p. 5.

${ }^{42}$ M. BRUSORIO, «L'intérêt légitime au changement de prénom », Gaz. Pal., 31 déc. 2005, n 365, p. 2.

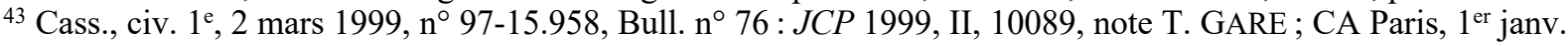
2002, AJ famille 2002. 108.
} 
Existant obligatoirement au jour où le juge statue ${ }^{44}, 1$ 'intérêt légitime sera retenu pour faire correspondre le prénom à un usage prolongé ${ }^{45}$, pour l'intégration de l'individu, ou en cas de prénom ridicule.

Concernant l'usage, l'inversion des prénoms d'un enfant a été autorisée pour permettre une harmonisation avec le prénom dont use l'intéressé et celui utilisé par les administrations publiques ${ }^{46}$ ou car le premier prénom était lié à un meurtre ${ }^{47}$. Plus étonnant, l'usage prolongé a permis à Barbara de devenir Babs ${ }^{48}$, ou à Marie de devenir Mariançon ${ }^{49}$.

Concernant l'objectif d'intégration, le changement peut être justifié quand le prénom de l'enfant est interdit par la loi du pays d'origine de ses parents et qu'il constitue un obstacle à ce que l'enfant se rende dans ce pays ${ }^{50}$. De même, l'intégration religieuse peut être prise en compte, permettant à Cherif Mohamed, souhaitant une complète assimilation à la communauté française, de devenir Michel $^{51}$. Plus généralement, le port d'un prénom pouvant renvoyer en apparence à une ethnie ou une religion peut justifier le changement de prénom, permettant à Isaac de devenir Jacques ${ }^{52}$ ou à David de s'appeler désormais Farid ${ }^{53}$.

Par ailleurs, il est nécessaire d'effacer tout risque de ricanements ou injures. Il en va ainsi d'un prénom jugé ridicule en soi, de sa faible cohérence avec l'apparence physique de celui qui le porte ${ }^{54}$, ou d'un prénom non conforme au genre de la personne en matière de transsexualisme, permettant à Wilfrid de devenir Chloé55. Par contre, le changement d'un prénom ne faisant objet ni de ricanements ni d'injures n'atteignant pas l'équilibre mental ou l'intégration sociale du porteur est refusé, tel principe justifie le refus de changer Judas en Maurice ${ }^{56}$.

Dans tous les cas, la persistance de l'intérêt légitime est nécessaire ${ }^{57}$, excluant les changements pour convenance personnelle ${ }^{58}$ ou successifs ${ }^{59}$. En ce sens, Zoubida, devenue Nadine, n'a pas pu reprendre son prénom d'origine ${ }^{60}$. Par contre, si les requêtes successives traduisent les étapes d'un itinéraire familial ou personnel difficile, les juges $\mathrm{du}$ fond vont permettre la modification. Tel fut le cas de Salah, devenue Claire lors de sa naturalisation, de reprendre son premier prénom afin d'obtenir un passeport tunisien et de ne pas être privée de ses droits successoraux en Tunisie ${ }^{61}$.

Cette brève observation de la jurisprudence démontre que le changement de prénom peut être un remède à la fantaisie des parents lorsque celle-ci, excessive, porte atteinte à l'intérêt de l'enfant. Cependant, ce remède est imparfait, notamment pour le mineur. En effet, la demande émanant de son représentant légal, c'est-à-dire le plus souvent de ses père et mère, le remède sera actionné par l'auteur de la fantaisie. En conséquence, le seul rempart à la fantaisie des parents semble plutôt passer par un renforcement du contrôle initial du choix des prénoms à la naissance. Pour cela, il peut être envisagé de redonner compétence à l'officier de l'état civil et, à l'instar de la Nouvelle-Zélande, d'établir une liste des prénoms interdits.

\footnotetext{
${ }^{44}$ Cass., civ. 1 ${ }^{\mathrm{e}}$, 6 mars 1990, Bull. n $62:$ D. 1990. 477, note J. MASSIP ; RTD civ. 1991. 449, obs. J. HAUSER.

45 Cass., civ. 1e 3 févr. 1981: D. 1981. 550, note J. MASSIP; RTD civ. 1981. 834, obs. R. NERSON et J. RUBELLIN-DEVICHI ; 10 oct. 1984, Bull. civ. I, n 255, p. 216 : Gaz. pal. 1985, 1, 187, note J. MASSIP ; CA Paris, 21 nov. $1995:$ D. 1996. 355, note T. GARE; 17 sept. $1996:$ D. 1996, somm. 384, obs. F. GRANET.

${ }^{46}$ CA Nancy, 24 sept. 2013, RG n ${ }^{\circ} 13 / 00867$ : RJPF 2013-12/7, obs. I. CORPART.

${ }^{47}$ CA Paris, 18 janv. 2013, RG n ${ }^{\circ} 12 / 09514$.

${ }^{48}$ CA Chambéry, 19 mars 2012 : RJPF 2014-2/16, obs. I. CORPART.

49 TGI 9 mars 2015 : AJ famille 2015. 296, obs. X. LABBEE.

${ }^{50}$ CA Versailles, 18 mai $2000:$ D. 2000 IR. 193.

${ }^{51}$ CA Paris, 20 déc. $1988: D .1989$. IR 37.

${ }^{52}$ Cass., civ. $1^{\mathrm{e}}$, 26 janv. 1965 : D. 195. 216 ; JCP 1965, II, 14064, concl. R. LINDON.

53 TGI Saumur, 3 mars $1977:$ JCP 1978, II, 18968, note T. P.

${ }^{54}$ CA Paris, 25 nov. 1993 : RTD civ. 1994. 322, obs. J. HAUSER.

${ }^{55}$ CA Rennes, 16 oct. $2012: D$. 2013. 156, note S. PARICARD.

56 TGI Nevers, 4 déc. 1974 : D. 1975. 567, note P. MALAURIE; RTD civ. 1975. 698, obs. R. NERSON.

${ }^{57}$ F. TERRE et D. FENOUILLET, Droit civil, op. cit., p. $195, \mathrm{n}^{\circ} 193$.

${ }^{58}$ Cass., civ. 1 1, 20 févr. 1996, Bull. civ. I, n 98 : Defrénois 1996, 985, obs. J. MASSIP ; RTD civ. 1996. 356, obs. J. HAUSER.

${ }^{59}$ CA Versailles, 27 mai 1999 : RTD civ. 1999. 598, obs. J. HAUSER.

${ }^{60}$ Cass. civ. 1re, 7 mars 1989 : D. 1989. IR. 105.

${ }^{61}$ CA Paris, 6 mai 2014 : RJPF 2014-7 et 8/25.
} 